\title{
Speed-Up of the Excited-State Benchmarking: Double-Hybrid Density Functionals as Test
}

\section{Cases}

Éric Brémond, ${ }^{* \dagger}$ Marika Savarese, ${ }^{\dagger}$ Ángel José Pérez-Jiménez, ${ }^{\ddagger}$ Juan Carlos Sancho-García, ${ }^{\ddagger}$ and Carlo Adamo,

†CompuNet, Istituto Italiano di Tecnologia, via Morego 30, I-16163 Genoa, Italy $\ddagger$ Departamento de Química Física, Universidad de Alicante, E-03080 Alicante, Spain ФChimie ParisTech, PSL Research University, CNRS, Institut de Recherche de Chimie Paris IRCP, F-75005 Paris, France

E-mail: eric.bremond@iit.it carlo.adamo@chimie-paristech.fr 


\begin{abstract}
The EX6-0, EX7-0 and EX7-1 representative benchmark sets are developed for the fast evaluation of the performance of a density functional, or more generally of a computational protocol, in modeling low-lying valence singlet-singlet excitation energies of organic dyes within the range of 1.5 to $4.5 \mathrm{eV}$. All sets share the advantage of being small (a maximum of 7 molecules), but providing statistical errors representative of larger and extended databases. To that extent, the EX7-1 benchmark set goes a step further and is composed by systems as small as possible in order to alleviate the associated computational cost. The reliability of all the sets is assessed through the benchmarking of 15 modern double-hybrid density functionals. The investigation shows not only that the 3 benchmark sets provide close error metrics for each density functional, but also that when taking advantage of the Resolution-of-the-Identity and a balanced triple- $\zeta$ basis set (e.g., def2-TZVP), double hybrids overperform the 'popular' hybrids in modeling vertical absorption, emission, and adiabatic energies.
\end{abstract}




\section{Introduction}

The accurate modeling of excited-state properties of large organic systems is a challenge of broad interest for chemists. From excited-state reactivity ${ }^{112}$ to electronic spectroscopy, $\frac{314}{\text { it }}$ allows for a comprehensive understanding of complex photomechanisms,,$[+5$ for the design and the characterization of novel candidates in dye and probe chemistry, ${ }^{8}$ and goes beyond qualitative aspects with the color simulation ${ }^{9}[1]$ or the quantification of side products for industrial purposes. $\frac{12[13}{12}$ This list is not exhaustive but representative of the various challenging excited-state applications explored within the modeling landscape.

The assessment of excited-state properties requires robust theoretical chemistry methods suitable for the type and complexity of the systems involved. Many of them exist, each one based on its own formalism (e.g., wavefunction, Green function, electron density) and each one providing different levels of accuracy and reliability. Among them, methods rooted in density-functional theory (DFT), 14 and particularly the adiabatic variant of time-dependent density-functional theory (TDDFT), $\frac{15}{15}$ are undoubtedly the most popular. Their popularity comes not only from their efficiency and their claim to routinely afford systems of hundred of atoms, but also from their continuous improvements through the development of new classes of exchange-correlation density-functional approximations. $\frac{16}{16}$ Those developments require tests and validations through standard and valuable benchmark sets built from robust reference energies/structures of systems representative of the targeted applications.

To this end, over the last ten years, numerous databases specifically dealing with excitedstate properties were developed. Valence, Rydberg and charge-transfer vertical excitations of small organic systems were the first properties to receive interest. 17 20 Their small size, ranging from 2 to 10 nonhydrogen atoms, add up the advantages to get a fast evaluation of the entire dataset and a robust theoretical reference. However their electronic spectroscopy signatures are mainly restricted to UV (wavelengths lower than $400 \mathrm{~nm}$ ), an energy domain of low interest for chemical applications. Later on, benchmark sets evolved to the probing of the UV-visible domain with extension to low-lying singlet-singlet $21+23$ and singlet-triplet 
vertical excitations $^{24}$ of large size organic chromophores, and a concomitant growing interest about adiabatic energy properties. $\frac{25+29}{2}$ Apart from benchmarks based on energy properties, some relevant excited-state structure and potential energy surface comparisons of organic systems exist but are still marginal. $\underline{30+32}$

Among the existing databases, Jacquemin et al. recently proposed a really large benchmark set of 80 large organic and organometallic systems that we denote here as RLex80 dataset. $^{25}$ It contains 80 molecules that are excellent candidates to probe for local and charge-transfer valence excitations in a broad UV-visible energy range $(\sim 1.5$ to $\sim 4.5 \mathrm{eV})$. However its complete assessment remains both computationally and time demanding since it requires the evaluation of 160 excited-state energies, 80 corresponding to ground-state minimum energy structures (absorption process) and 80 others corresponding to excited-state relaxed energy structures (emission process). As a result using such a dataset to train or to validate the development of a new theoretical method is indeed statistically relevant but it is also technically cumbersome.

In this article, we propose a robust and computationally inexpensive way to probe new theoretical developments for excited-state properties by deriving small representative subsets of the large RLex80 benchmark set. Taking inspiration from pioneering works by Truhlar and coworkers in which they specifically derive subsets from large and diverse databases (i.e., atomization energies of main-group 33 and transition metal-based systems, $\frac{34 \sqrt[35]{35}}{\text { barrier }}$ heights of reactions $\left.{ }^{36}\right)$, we construct here 3 equivalent small benchmark sets guaranteeing the chemical diversity and the statistical representativity of the large RLex80 dataset with the aim to get a fast and robust idea of the excited-state performance of a method. More precisely, the 3 small sets are built under 3 successive constraints imposed (i) to conserve the standard statistical criteria; (ii) to conserve the broad range of reference values of the large RLex80 dataset; (iii) to drastically reduce the computational power needed to assess them. These small sets are named as part of what we call the $\operatorname{EXN}$ family (i.e., EX60, EX7-0, EX7-1) and are especially designed to measure the performance of a theoretical 
method to model vertical absorption, vertical emission and adiabatic energies of local and charge-transfer character.

Finally, the EX $N$ family of benchmark sets is then used to test the performance of a panel of 15 modern double-hybrid density functionals. Their computational cost is larger than the one provided by conventional DFT methods, thus representing a natural application to the EX6-0, EX7-0 and EX7-1 benchmark sets. Pioneering investigations already showed that some of these density functionals are quite promising to model excited-state properties but

their extended benchmarking and related applications are still scarce in the domain. $21 / 22137+39$ We propose here to fill the gap by assessing a large number of double hybrids, including parameterized as well as parameter-free approaches, for excited-state purposes.

\section{Theoretical Background}

Introduced in $2006, \frac{40}{4}$ the modern variant of double hybrids (DH) expresses the exchangecorrelation energy as the sum of semilocal density functional approximation (DFA) and fully nonlocal energy terms such as

$$
E_{x c}^{\mathrm{DH}}=a_{x} E_{x}^{\mathrm{EXX}}+\left(1-a_{x}\right) E_{x}^{\mathrm{DFA}}+\left(1-a_{c}\right) E_{c}^{\mathrm{DFA}}+a_{c} E_{c}^{\mathrm{PT} 2},
$$

where $a_{x}$ and $a_{c}$ governs the mixing between the nonlocal exact-like exchange (EXX) and the nonlocal correlation computed by second-order perturbation theory (PT2), respectively. The first three terms belongs to the well known global-hybrid (GH) expression

$$
E_{x c}^{\mathrm{GH}}=a_{x} E_{x}^{\mathrm{EXX}}+\left(1-a_{x}\right) E_{x}^{\mathrm{DFA}}+\left(1-a_{c}\right) E_{c}^{\mathrm{DFA}}
$$

and are computed in a self-consistent fashion. The last energy term is evaluated a posteriori from the GH self-consistently optimized set of Kohn-Sham orbitals and eigenvalues, and then added to the GH exchange-correlation energy prior being weighted by the $a_{c}$ coefficient. 
The resulting DH expression is nowadays interpreted by numerous authors $\frac{1614143}{4 n}$ in a parameter-free (fully parameterized) fashion by nonempirically (empirically) setting the $\left\{a_{x}, a_{c}\right\}$ mixing coefficients, and by carefully choosing the semilocal approximation. Despite of their divergent roots, both approaches lead to similar conclusions in terms of ground-state property performance, $\frac{44}{4}$ and constitute a large performance improvement with respect to parent density functionals belonging from the semilocal or hybrid approximations. $\underline{45}$

The extension of double-hybrid density functionals to excited states reproduces the twostep fashion protocol described for the ground state. ${ }^{39}$ The GH vertical excitation energy $\left(\Omega_{\mathrm{GH}}\right)$ which derives from the $\mathrm{GH}$ exchange-correlation equation (eq 2$)$, is estimated from the Tamm-Dancoff approximation $\underline{46}$ (TDA) of the linear-response TDDFT equations. $\frac{15}{15}$ They states that

$$
\mathbf{A X}=\Omega_{\mathrm{TDA}-\mathrm{GH}} \mathbf{X}
$$

with $\mathbf{A}$ and $\mathbf{X}$ representing the Hamiltonian matrix excitation matrix and the corresponding excitation vector, respectively.

By analogy with the ground state, eq 3 is a CIS-like expression which can be corrected following a second-order perturbation approach. $\frac{47}{7}$ The resulting CIS(D)-like correction $\left(\Delta_{(\mathrm{D})}\right)$ is finally summed up with the GH vertical excitation energy to give the DH vertical excitation energy such as:

$$
\Omega_{\mathrm{TDA}-\mathrm{DH}}=\Omega_{\mathrm{TDA}-\mathrm{GH}}+a_{c} \Delta_{(\mathrm{D})}
$$

where the $a_{c}$ coefficient corresponds to the one set by eq 1 and denotes the fraction of CIS(D)-like correction.

\section{Subset Derivation}

The RLex80 dataset is a robust statistical database (see Figure S1 in the Supporting Information for a graphical representation of the molecules) containing 80 organic systems 
whose structures are optimized in their ground- and low-lying excited-state, thus allowing the comparison with the corresponding experimental 0-0 excitation energies. Experimental measurements are among the best references to build a benchmark set yet they are also known to be condition-dependent and in the present case, prone to solvent and vibrational effects. We prefer here to turn to a theoretical reference in lieu of comparing equivalent quantities and avoiding accumulation of errors. In particular, the $\mathrm{CC} 2$ second-order approximate coupled cluster method has been adequately chosen previously to be the reference approach for accurate spectroscopic properties of large organic chromophores, ${ }^{21}$ and to obtain accurate reference energies within the RLex80 dataset. ${ }^{25}$

This choice strongly increases the number of reference energy values from 80 to 240 (i.e., 80 vertical absorption, 80 vertical emission and 80 adiabatic energies), improving the statistical robustness of the benchmark set, but preventing form its fast assessment, a criteria often requested during the development of a novel computational protocol. In order to circumvent this problem, we propose here to derive some statistically relevant subsets from the RLex80 dataset following a reliable and efficient initiative firstly described by Lynch and Truhlar. $\frac{33}{33}$ Within this framework, the statistical agreement between the parent dataset and its subset implies a close equality of the error measures (vide infra) of both sets: the mean signed deviation (MSD), the standard deviation (STD) and the mean absolute deviation (MAD). These metrics are widely employed, $\stackrel{48}{\frac{48}{4}}$ and are thus used in the following to discriminate the newly designed subsets.

More precisely, the design procedure followed here compares the error measures over the adiabatic energies of the parent RLex80 dataset with the ones of its subsets for a selection large enough of 16 TDDFT-based computational protocols (see Table S1 in the Supporting Information). The methods are chosen to represent the exchange-correlation densityfunctional landscape developed over the past 20 years, i.e., density functionals spanning from pure to hybrid and double-hybrid approximations. To determine and validate the most statistically representative subset $\left(\mathrm{S}_{N}\right)$ of $N$ systems, the 16 selected computational protocols 
are split into a training and a test set named TrainXC8 and TestXC8, respectively. During the training step, TrainXC8 feeds a normalized absolute error function ERR:

$$
\operatorname{ERR}\left(\mathrm{S}_{N}\right)=\frac{\sum_{i=1}^{8} \sum_{m_{i}=\left\{\mathrm{MSD}_{i}, \mathrm{MAD}_{i}, \mathrm{STD}_{i}\right\}}\left|m_{i}\left(\mathrm{~S}_{N}\right)-m_{i}(\mathrm{RLex} 80)\right|}{\sum_{i=1}^{8} \sum_{m_{i}=\left\{\mathrm{MSD}_{i}, \mathrm{MAD}_{i}, \mathrm{STD}_{i}\right\}}\left|m_{i}(\mathrm{RLex} 80)\right|},
$$

which calculates the absolute error between a statistical criteria $m_{i}$ estimated from the RLex80 dataset and its subset $S_{N}$ for the $i$ th computational protocol ( $i$ runs over 1 to 8 in case of TrainXC8). For each subset size $N \in[1,80], N$ systems are chosen to minimize the ERR function such as:

$$
\operatorname{EXN}:=\min _{\mathrm{S}_{N}}\left\{\operatorname{ERR}\left(\mathrm{S}_{N}\right)\right\} .
$$

The minimization procedure returns the EXN subset which is the most statistically representative subset of $N$ systems of the RLex80 dataset. According to eq 5 we can expect that, the larger $N$ is, the better the statistical agreement between the RLex80 dataset and its subset will be. As a consequence, the choice of $N$ is a trade-off between deviations in reproducing the statistical measures and the computational time.

The training step is followed by an assessment step which is typically afforded to validate the robustness of the statistical treatment, or in other words, to verify that the EXN subset provides similar values of the ERR function (eq 5) when fed by the computational protocols included into both TrainXC8 and TestXC8. In addition, the assessment step is also supplemented by 4 post-Hartree-Fock approaches defined in TestWF4 (see Table S1 in the Supporting Information). In this sense, the validation extends the application range of the EXN subset to wavefunction-based methods. Note that the subset derived under this procedure will be dubbed as EX6-0 (vide infra).

As mentioned above, the subset derivation based on the training and assessment loop is mainly based on pure statistical arguments. The potential risk of this process is the lack 
of chemical diversity which reflects in the large range of excitation energies contained into the RLex80 dataset. This diversity is particularly important to judge the quality of density functionals since the range of errors strongly depends on the character of the excitation (see Figure S2 in the Supporting Information). Therefore, to conserve this chemical feature, a further constraint is added to the minimization process, ultimately leading to the EX7-0 benchmark set. It consists in conserving the broad range of reference excitation energies of the RLex80 dataset by partitioning it according to a one-dimensional (1D) histogram approach where the number of bins is optimally tuned following the Freedman-Diaconis rule. ${ }^{49}$ This partition fixes automatically the number $N$ of systems included into the subset as equal to the number of bins of the histogram. As a result the newly derived subset is not only statistically alike the RLex 80 dataset, but it also shares in proportion its chemical diversity.

However, note that the question of the computational cost still remains. Deriving a small subset of the large RLex80 dataset contributes to a general decrease of the overall CPU time spent to assess the entire set, but it does not directly impact on the computational power needed by any software to afford the excited-state evaluation of a single system. A pragmatic remedy to further reduce the computational cost consists in adding another constraint during the subset derivation, that will finally yield the EX7-1 set of systems. This constraint is based on the size of the system and more particularly on its number of electrons. Consequently, the 80 systems of the RLex80 dataset are partitioned into a two-dimensional (2D) histogram (broad range of reference energies, total number of electrons) in which the bin size of each dimension is again determined by the Freedman-Diaconis rule. $\stackrel{49}{\text { During }}$ the statistical minimization of the ERR function (eq 5), one system is selected in each bin along the reference energy axis, constraining the selection to the lowest bin along the 'total number of electrons' axis. As a result the newly derived subset shares not only the chemical diversity and the statistical likeness with the RLex80 dataset, but it also tends to reduce concomitantly the computational time and cost during its assessment. 


\section{Computational Details}

All the DFT and TDDFT computations were performed on the fully optimized ground- and excited-state structures belonging to the RLex80 dataset and reported in Reference 25. The the adiabatic energy is calculated as the difference between the excited- and ground-state energies of the relaxed excited- and ground-state structures, respectively, while the vertical absorption (emission) is computed as the difference between the excited- and ground-state energies of the relaxed ground-state (excited-state) structure.

In particular, for the pure and hybrid density functionals involved in the TrainXC8 (e.g.,

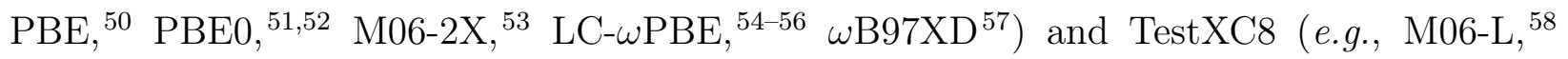

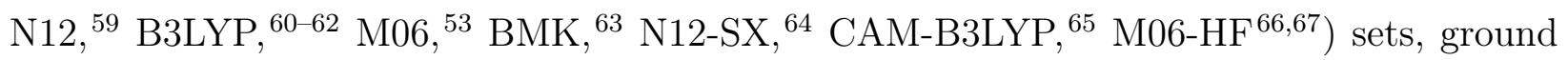
and excited-state energies were estimated within the Kohn-Sham DFT and linear-response TDDFT frameworks, respectively. In relation with these families of density functionals, the $6-31+\mathrm{G}(d)$ double- $\zeta$ basis set ${ }^{68 \mid 69}$ which is recommended for a fast and meaningful treatment of valence excitations, $\frac{170}{}$ was used. The heavy metal atoms were treated with the LanL2DZ basis set and the corresponding effective core potentials. $\frac{7172}{172}$ All these computations were performed with the Gaussian'09 program ${ }^{[73}$ using tight SCF convergence criteria and ultrafine integration grids.

All the double-hybrid-based computations were performed with the Orca software ${ }^{74}$ (release 3.0.3) using a tight SCF convergence criteria and taking advantage of the 'resolution-ofthe-identity' (RI) approximation: the 'chain-of-spheres' algorithm ${ }^{[75]}$ (COSX) was turned on to efficiently and accurately treat the exact-like exchange contribution in self-consistent procedures. ${ }^{[76}$ To nearly reach the basis set convergence (see Figure S3 in the Supporting Information), the large Ahlrichs def2-TZVP triple- $\zeta$ basis set ${ }^{177}$ together with its def2-TZVP $/ J K$ and def2-TZVP $/ \mathrm{C}$ auxiliary counterparts were also used. In the case of dealing with heavy metal atoms, the corresponding Stuttgart-Dresden effective core potentials were added to the computational procedure. $\stackrel{\square 7 / 78}{ }$

Note that the requested excitation energies obtained at the aug-cc-pVTZ level of the- 
ory $\frac{7980}{8}$ with the post-Hartree-Fock methods of the TestWF4 set (i.e., ADC(2),,$\frac{81}{\text { SOS- }}$

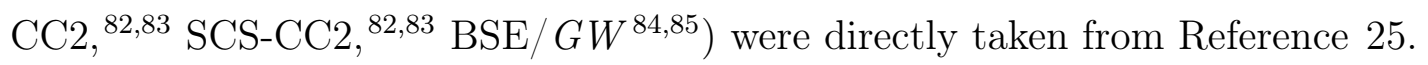

\section{Results and Discussion}

\subsection{EX6-0: a subset statistically alike the RLex80 dataset}

The first subset derivation is performed without applying any specific constraint on the statistical minimization procedure. It just consists in selecting molecules from the RLex 80 dataset to build a subset made with $N \in[1,80]$ element(s) and whose statistical performance criteria (e.g., MSD, MAD, STD) are similar to those of the parent set by minimizing the ERR function (eq 5). Figure 1 plots the evolution of the ERR function versus the size $N$ of the subset. It especially shows how the increase of $N$ leads to a fast decrease of the ERR function when going from $N=1(\mathrm{ERR}=29.4 \%)$ to $N=6(\mathrm{ERR}=2.5 \%)$.

Fixing $N$ is merely a question of compromise between the reproduction of the MSD, MAD and STD measures and the computational time employed. As a general trend, vertical transition energies are estimated within errors of the order of $\sim 2 \times 10^{-1} \mathrm{eV}$ for global-hybrid density functionals. ${ }^{86} \mathrm{~A}$ bias of $2.5 \%$ on this measure gives a variation of $\sim 5 \times 10^{-3} \mathrm{eV}$, which is below the numerical accuracy of the CC2 energies taken in Reference. ${ }^{25}$ For this reason, we establish a $2.5 \%$ threshold on the ERR function as convergence limit and set the optimal subset size as $N=6$.

The corresponding subset is consequently named EX6-0. Its molecules are depicted in Figure 2 and their spectroscopic features are provided in Table 1 . From a spectroscopic point of view, these systems are very different since the character of their first low-lying transition can be of local- $\left(\pi \rightarrow \pi^{\star}\right)$ or charge-transfer-type with an overall range of energies spanning between 2.02 and $4.56 \mathrm{eV}$. This wide range of energies gets closer to the one of the RLex80 dataset $(1.71 \mathrm{eV}$ to $4.56 \mathrm{eV})$ also guaranteeing a parent chemical diversity.

Figure 3 shows the correlation between MADs from the large RLex80 dataset and the 
newly developed EX6-0 subset for the TrainXC8 and TestXC8 sets. More details about the MSD and STD measures are provided in Figure S4 in the Supporting Information. As a general trend, the linear agreement is respected for each statistical measure with very small deviations with respect to the diagonal, always lower than $0.02 \mathrm{eV}$. Thus the EX6-0 subset is an excellent approach to evaluate the performance of a given density functional (from pure to hybrid and double-hybrid approximations) in modeling the low-lying excited-state properties of organic dyes. Figure 3 confirms also that the applicability of the subset can be successfully extended to the post-Hartree-Fock methods included into TestWF4.

\subsection{EX7-0: a subset statistically alike and respecting the chemical diversity of the RLex80 dataset}

The second subset derivation aims to go beyond the systematic statistical minimization procedure fixed by eq 6 while imposing a constraint which ensures the recovery of the chemical diversity of the RLex80 dataset. This constraint consists in conserving its wide and broad range of excitation energies (from $1.71 \mathrm{eV}$ to $4.56 \mathrm{eV}$ ). This feature is particularly important for some families of exchange-correlation density functionals for which the error estimating the excitation energies increases when the latter increases (see Figure S2 in the Supporting Information). The constraint is applied by partitioning the wide range of excitation energies according to a 1D-histogram approach. The number of bins is optimally estimated by the Freedman-Diaconis rule and is found equal to 7 (bin width of $\sim 0.41 \mathrm{eV}$ ), fixing automatically the size of the newly derived subset as $N=7$.

Once the energy partitioning is done, the selection of the 7 elements is performed by choosing a system in each bin of the 1D-histogram and minimizing the ERR function (eq

5). An illustration of the selection procedure is provided in Figure 4. The ERR function is finally minimized up to $5.7 \%$ with an excitation range spanning between 1.89 and $4.56 \mathrm{eV}$, and the newly derived subset is named EX7-0. By direct comparison with the EX6-0 subset, EX7-0 includes one molecule more and its chemical diversity is by construction closer to the 
one of the RLex80 dataset. However its statistical agreement remains larger $(5.7 \%$ instead of $2.5 \%$ for the EX6-0 subset) but still acceptable.

The molecules included into the EX7-0 subset are sketched in Figure 5 and their respective spectroscopic features are reported in Table 2. Here again, the chemical diversity is rich and composed by low-lying excitations of local- $\left(\pi \rightarrow \pi^{\star}\right)$ or charge-transfer-type characters, an important feature to benchmark the performance of density-functional approximations. Among the 7 compounds, two molecules overlap between EX6-0 and EX7-0. The first one is a push-pull donor- $\pi$-acceptor chromophore made from the triphenylamine and thiazol moities (4) , with a low-lying transition keeping a notably charge-transfer character. The second one is biphenyl ( $\underline{\mathbf{0 1}})$, a common moiety of many organic compounds.

The correlations between MADs from the large RLex80 dataset and the newly developed EX7-0 subset for the computational protocols defined in the TrainXC8 and TestXC8 sets are reported in Figure 3. More details about the MSD and STD measures are provided in Figure S5 in the Supporting Information. MADs calculated for the $8+8$ computational protocols agree with small deviations to the diagonal up to $0.04 \mathrm{eV}$. A similar behavior is observed while switching from DFT to post-Hartree-Fock methods of the TestWF4 set. Thus the EX7-0 subset is another faithful benchmark subset to judge the quality of a computational approach in modeling the low-lying excited-state properties of organic dyes.

\subsection{EX7-1: a computationally costless variant of the EX7-0 subset}

Generally speaking, the subset derivation already reduces the overall computational time but here, we go a step further by tackling the topic of the computational cost through the addition of 'a system size' constraint based on the total number of electrons per molecule. It consists in partitioning the 80 systems according to a 2D-histogram approach where the

first and second axis correspond to (i) the range of excitation energies and (ii) the number of electrons, respectively. The bin sizes along each axis are automatically tuned according to the Freedman-Diaconis rule and by analogy to the previous procedure, the newly designed 
subset carries $N=7$ elements to conserve the chemical diversity feature.

Like in the previous approach, the 7 compounds are selected in the 7 bins along the first axis but restricting the choice to the lowest populated bins along the second axis. Figure 6 depicts the 2D-partitioning and the 'red line' follows the 2D-bins from which are selected the molecules. The ERR function minimization procedure (eq 6) finalizes the selection. The minimum value of ERR is found to be $17.4 \%$ and the derived subset is named EX7-1.

The molecules included into the EX7-1 subset are represented in Figure 7 and their respective spectroscopic features are reported in Table 3. The chemical diversity is still rich and composed by low-lying excitations of local- $\left(\pi \rightarrow \pi^{\star}\right)$ or charge-transfer-type characters, but the size of the systems involved dramatically decreased. It spans now between 82 and 137 electrons (22 and 32 atoms, respectively) while in the other subsets, the largest system has 182 electrons (48 atoms) in the case of EX6-0, and 212 electrons (49 atoms) for EX7-0. By reference to EX7-0, this new restriction constitutes a total economy of 361 electrons (80 atoms) which represents a significant gain in term of computational cost. However, it should be pointed out that this economy of atoms could restrict the nature of the electronic transitions involved, since long-range charge-transfer excitations are no longer considered. As a consequence, this restriction reduces somehow the chemical diversity of the subset.

The correlations between MADs estimated from the RLex80 dataset and its EX7-1 subset for the computational protocols defined in the TrainXC8 and TestXC8 sets are reported in Figure 3. More details about the MSD and STD measures are provided in Figure S6 in the Supporting Information. The deviation to the diagonal for those statistical criteria is one order of magnitude more $(\sim 0.10 \mathrm{eV})$ with respect to the ones found for EX6-0 and EX7-0. This larger deviation is explained by the successive constraints applied to derive the subset. They considerably restrict the pool of systems and consequently worsen the statistical agreement. Surprisingly, the deviation to the diagonal is lower for the postHartree-Fock approaches of the TestWF4 set $(\sim 0.06 \mathrm{eV})$. Nevertheless this less satisfactory statistical agreement does not discredit EX7-1 since again the main objective is to propose a 
subset affordable by computational approaches that are more resource-and-time-consuming than TDDFT.

\subsection{Computational effort to assess the $\mathrm{EX} N$ family of subsets}

The advantages and inconveniences of each benchmark set of the EXN family, in terms of computational time and cost, is now carefully analyzed. The assessment is performed with the PBE-QIDH parameter-free double-hybrid density functional. PBE-QIDH, and more generally double hybrids, are excellent probing examples for this investigation since their formal computational cost is larger than the one of more standard global or range-separated hybrids $\left(O\left(N^{5}\right)\right.$ versus $O\left(N^{3}\right)$ with $N$ the number of electrons of the system), but strongly alleviated by density-fitting techniques.

Figure 8 reports the CPU time trends obtained for the EX6-0, EX7-0 and EX7-1 subsets. With respect to the parent RLex80 dataset, the total computational time to assess an entire subset is decreased by a factor 100 and varies in proportion between 1.5 and $8.5 \%$ (EX7-1 and EX7-0, respectively). EX6-0 is in between with a total CPU time ratio of $4.7 \%$.

The time performance is especially emphasized for EX7-1 which is designed to reduce the computational cost. Its mean CPU time $(0.2 \%)$ is a few times smaller than that of EX6-0, despite including one system morer. The relevance of EX7-1 is not only related to the overall computational CPU time, but also to the time spent to assess the largest system, a bottleneck often found in standard computations. It is estimated to be about $0.5 \%$ for EX7-1, while it is about 1.3 and $2.8 \%$ for EX6-0 and EX7-0, respectively. As a result the EX7-1 subset is not only suitable for TDDFT benchmarking but also for the more resource-demanding and time-consuming computational approaches.

Overall, the impressive resource gain added to the excellent physical and chemical representativity of the EX6-0, EX7-0 and EX7-1 subsets turn them into excellent alternatives to the large RLex80 dataset when benchmarking any kind of computational protocols for excited-state purposes. 


\subsection{Double-hybrid density functionals as probing examples for the EX $N$ family of subsets}

The double-hybrid class of density-functional approximations is of course computationally more demanding than the 'popular' hybrid one, but it is worth to recall that climbing from singly to doubly hybridized approximation brings the considerable advantage to drastically improve the performance of density-based methods for any kinds of ground-state energy $\underline{\underline{45}}$ and structure properties. ${ }^{87}$ Regarding the topic of systems relevant for excited-state chemical applications, the performance assessment of double-hybrid density functionals is still $\operatorname{rare}^{2122137 \sqrt[39]{3}}$ and lets wonder if there is still room for this family of approximation. The RLex80 dataset gives here the opportunity to obtain an extended view of their energy performance taking gas-phase $\mathrm{CC} 2$ energies as reference.

As a general rule of thumb, the increase of the fraction of exact-like exchange tends already to decrease the error on vertical absorption energies (Figure 9). This is especially true for the sequence PBE (EXX: 0\%; MAD: $0.66 \mathrm{eV}$ ), B3LYP (EXX: 20\%; MAD: 0.30 $\mathrm{eV}$ ), and BMK (EXX: 40\%; MAD: $0.16 \mathrm{eV}$ ) on the whole RLex80 dataset. Then casting a fraction of nonlocal CIS(D)-like correction contributes to further decrease these deviations. Indeed in going from B3LYP (PBE0) to B2-PLYP (PBE-QIDH) improves the absolute error from $0.30(0.22) \mathrm{eV}$ to $0.10(0.22) \mathrm{eV}$ (Figure 9). This general trend shows not only that the double-hybrid approximation improves the quality of the excited-state energy estimations but also that the performance improvement depends on the underlying density functional. $\stackrel{42}{4}$

To get a representative flavor of the performance spreading of the different variants of double-hybrid density functionals, we select 15 of them (Table 4) to assess the EX6-0, EX70 and EX7-1 benchmark sets developed below. Such an assessment will not only provide new insight regarding density functional benchmarking but will show how the performance varies going from one representative subset to another. Figure 10 reports the MAD statistical measures for the three subsets over vertical absorption, vertical emission and adiabatic 
energies taking gas-phase CC2 computations as reference. CIS(D) is added for the sake of comparison.

Concerning vertical absorption energies, the MADs estimated on the EX6-0 subset span between 0.08 and $0.44 \mathrm{eV}$ for B2IP-PLYP and B2-PPW91, respectively. Only B2-PPW91 performs worse than CIS(D) $(0.35 \mathrm{eV})$ for this property. This poor behavior regarding excited-state properties is quite surprising $\frac{\sqrt{88}}{}$ since the latter double hybrid is specifically optimized to reproduce dipole polarizability and hyperpolarizability properties. It is probably linked to the too restricted choice of systems involved in its parameterization loop and to the strong lack of exact-like exchange ( $20 \%$ instead of more than $50 \%$ for the others). Despite of this singularity, double hybrids are in line (for some of them) and even better (for others) with global hybrids for such a property (Figures 9 and 10 ). As a result, they can be viewed as an excellent alternative to standard global hybrids to model accurately low-lying singlet-singlet vertical absorption energies. This performance trend is also confirmed by assessing the EX7-0 and EX7-1 subsets (Figures 9 and 10). Despite of the chemical diversity constraint, EX7-0 reproduces faithfully the MADs estimates of vertical absorption energies obtained from the EX6-0 with an error all the time lower than $0.02 \mathrm{eV}$. For EX7-1, the largest deviation remains lower than $0.10 \mathrm{eV}$.

For vertical emission energies, double hybrids (Figure 10) perform as well as vertical absorption with variations (often negative) of about $\sim 10^{-2} \mathrm{eV}$ observed on the 3 subsets. This behavior is not specific to double hybrids. It is general to all tested exchange-correlation density functionals and post-Hartree-Fock methods (see Figure S7, S8 and S9 in the Supporting Information). The performance similarity for vertical absorption and emission processes is also confirmed by the close deviations found between the 3 subsets. Here again, the largest error between EX6-0 and EX7-0 (EX7-1) is lower than $0.02(0.10) \mathrm{eV}$.

Moving to adiabatic energy properties slightly worse the performance of double hybrids with respect to vertical processes (Figure 10). On the EX6-0 subset, the MADs span between $0.10 \mathrm{eV}$ and $0.33 \mathrm{eV}$ for B2-PLYP and PBE-QIDH, respectively. Extending the performance 
assessment to the EX7-0 and EX7-1 subsets brings similar ranges of errors. The origin of the performance decrease between vertical and adiabatic properties comes from the structure reorganization between ground and excited state which adds another source of error. At the moment, this error is difficult to quantify and is still matter of ongoing research since analytical gradients for time-dependent double-hybrid approaches are missing and the research of minimum excited-state energy structures derived from robust reference protocols are still computationally too demanding.

The 15 double-hybrid density functionals investigated here prove that the EX6-0, EX7-0 and EX7-1 representative subsets of the large RLex80 dataset are able to provide a very close statistical agreement between themselves. As a consequence, they can be viewed as an excellent and fast alternative to the RLex80 dataset keeping in mind that EX6-0 provides a better statistical agreement with RLex80 than EX7-0 and EX7-1, but EX7-1 is faster to assess than EX6-0 and EX7-0. Moreover, the 15 double-hybrid density functionals show also that most of them are able to overperform global and range-separated hybrids for such a job. This improvement is explained by the addition of a fraction of CIS(D)-like nonlocal correlation whose the amount rangs between 10 (B2-PPW91) and 50\% (PBE0-2). The role of this contribution is to compensate the main faults of the hybrid part, so that the nonlocal CIS(D)-like contribution prevents the overestimation of excitations energies. ${ }^{42}$

In order to point out this effect, Figure 11 represents the correlation between the MADs obtained with and without the CIS(D)-like contribution for 3 double hybrids, namely PBE0DH, B2-PLYP and PBE-QIDH. Overall, it appears that the casting of this nonlocal component improves quasi-systematically the estimation of vertical absorption, emission and adiabatic energies. However, the magnitude of the correction differs and is not only linked to the fraction of CIS(D)-like nonlocal correlation. On one hand, PBE0-DH and B2-PLYP cast similar fractions of exact-like exchange (50 and 53\%, respectively) and different amounts of CIS(D)-like correction (12.5 and 27\%, respectively). Deviations obtained with the former are closer to the diagonal than the ones computed with the latter. On the other hand, an 
intermediate behavior is observed for PBE-QIDH ( $\sim 33 \%$ of CIS(D)-like correction) which shares the same parent PBE functional with PBE0-DH but a larger fraction of exact-like exchange $(\sim 69 \%)$. While these trends deserve a deeper investigation, they suggest a not straightforward rationalization in term of exact-like exchange and CIS(D)-like nonlocal correlation. There is still room for the assessment of excited-state properties with double-hybrid density functionals.

\section{Conclusions}

We develop here the EXN family of small datasets for the fast assessment of computational protocols in modeling excited-state properties of challenging dyes. The datasets are designed based on the low-lying valence excitations of organic molecules which are at the origin of the main features of their absorption and emission spectra. Named as EX6-0, EX7-0 and EX7-1, they combine the statistical representativity of the RLex80 collection of organic and organometallic compounds which is nowadays taken as a reference for probing the UV/vis excited-state signatures of dyes. We further emphasize the following key features of the subsets:

1. The EX6-0 dataset contains only 6 systems selected to accurately reproduce mean signed, mean absolute and standard deviations criteria of the large RLex80 dataset.

2. The EX7-0 dataset is slightly larger. Its 7 systems are selected to conserve the largest range of excitation energies, and to accurately keep a statistical agreement with the large RLex80 dataset.

3. The EX7-1 dataset contains also 7 systems selected this time to conserve the largest range of excitation energies and the smallest number of electrons, still keeping a statistical agreement with the large RLex80 dataset. 
To illustrate the efficiency of the EXN family of datasets, we selected a large panel of modern double-hybrid density functionals as working examples. By comparison with the reference RLex80 set, we show that the CPU time gain to assess an entire EX $N$ dataset can decrease up to $1.5 \%$. We show also that the derived datasets provide statistical measures (e.g., MSD, MAD, STD) very close to RLex80, with deviations found in average as marginal and significantly smaller than deviations with respect to the reference excitation energies. Above all, we prove that double-hybrid density functionals emerge as an interesting alternative to global and range-separated hybrids for the evaluation of low-lying valence excitations with local or charge-transfer character with mean absolute deviations reaching up to 0.10 $\mathrm{eV}$ for vertical absorption, emission and adiabatic energies. We demonstrate that such a performance is fairly obtained by using a balanced triple- $\zeta$ basis set (e.g., def2-TZVP) and by taking advantage of the Resolution-of-the-Identity which strongly alleviates the computational cost. We also conclude that this excellent performance is not only due to the large fraction of exact-like exchange casted into double hybrids but also to the CIS(D)-like correction, a component which pushes TDDFT beyond the modeling of single-excitation character. 


\section{Acknowledgement}

AJPJ and JCSG thank the "Ministerio de Economía y Competitividad" of Spain and the "European Regional Development Fund" through the project CTQ2014-55073-P for financial support. 
Table 1: List of the systems included into the EX6-0 subset. Reference vertical absorption (abs.; in eV), vertical emission (emi.; in eV) and adiabatic (adi.; in eV) energies computed in gas phase at $\mathrm{CC} 2$ level of theory are reported. All the computations are done with the aug-cc-pVTZ basis set.

\begin{tabular}{ccccc} 
system & type & abs. $^{a}$ & emi. $^{a}$ & adi $^{a}$ \\
\hline$\underline{\mathbf{2 6}}$ & iminium dye & 2.48 & 2.35 & 2.37 \\
$\mathbf{5 4}$ & thiophene dye & 2.42 & 2.19 & 2.27 \\
$\mathbf{5 9}$ & CT dye & 2.66 & 2.18 & 2.31 \\
$\mathbf{\underline { \mathbf { 2 } }}$ & styrene dye & 3.53 & 3.01 & 3.15 \\
$\mathbf{\mathbf { 7 4 }}$ & CT dye & 3.71 & 3.21 & 3.32 \\
$\underline{\mathbf{0 1}}$ & hydrocarbon & 5.41 & 4.33 & 4.72 \\
${ }^{a}$ from Reference 25. & & &
\end{tabular}


Table 2: List of the systems included into the EX7-0 subset. Reference vertical absorption (abs.; in eV), vertical emission (emi.; in eV) and adiabatic (adi.; in eV) energies computed in gas phase at $\mathrm{CC} 2$ level of theory are reported. All the computations are done with the aug-cc-pVTZ basis set.

\begin{tabular}{ccccc} 
system & type & abs. $^{a}$ & emi. $^{a}$ & adi. $^{a}$ \\
\hline $\mathbf{0 8}$ & keto dye & 2.24 & 1.91 & 1.94 \\
$\underline{\mathbf{5 2}}$ & thiophene dye & 2.65 & 2.22 & 2.35 \\
$\mathbf{\mathbf { 7 5 }}$ & keto dye & 3.37 & 2.79 & 2.87 \\
$\mathbf{\mathbf { 7 4 }}$ & CT dye & 3.71 & 3.21 & 3.32 \\
$\mathbf{\mathbf { 3 3 }}$ & complex dye & 4.06 & 3.47 & 3.73 \\
$\underline{\mathbf{4 1}}$ & hydrocarbon & 4.77 & 4.18 & 4.37 \\
$\mathbf{0 1}$ & hydrocarbon & 5.41 & 4.33 & 4.72 \\
${ }^{a}$ from Reference $\underline{\mathbf{2 5} .}$ & & &
\end{tabular}


Table 3: List of the systems included into the EX7-1 subset. Reference vertical absorption (abs.; in eV), vertical emission (emi.; in eV) and adiabatic (adi.; in eV) energies computed in gas phase at $\mathrm{CC} 2$ level of theory are reported. All the computations are done with the aug-cc-pVTZ basis set.

\begin{tabular}{ccccc} 
system & type & abs. $^{a}$ & emi. $^{a}$ & adi. $^{a}$ \\
\hline$\underline{\mathbf{2 6}}$ & iminium dye & 2.48 & 2.35 & 2.37 \\
$\mathbf{1 6}$ & CT dye & 2.53 & 1.85 & 2.13 \\
$\mathbf{3 4}$ & polyaromatic & 3.13 & 2.70 & 2.80 \\
$\mathbf{1 2}$ & keto dye & 4.05 & 3.40 & 3.65 \\
$\mathbf{0 3}$ & keto dye & 4.27 & 3.34 & 3.87 \\
$\underline{\mathbf{4 1}}$ & hydrocarbon & 4.77 & 4.18 & 4.37 \\
$\underline{\mathbf{0 1}}$ & hydrocarbon & 5.41 & 4.33 & 4.72 \\
${ }^{a}$ from Reference 25. & & &
\end{tabular}


Table 4: List of double-hybrid density functionals ordered by their increasing exact-like exchange weight, and wave function theory-based methods considered in this work. EXX and PT2 denote the percentage of exact-like exchange and second-order perturbation correlation, respectively.

\begin{tabular}{ccccccc} 
Name & Year & Type $^{a}$ & \% EXX & \% PT2 & & Reference(s) \\
\hline B2-PPW91 & 2012 & DH-GGA & 20 & 10 & 89 \\
PBE0-DH & 2011 & DH-GGA & 50 & 13 & 90,91 \\
TPSS0-DH & 2011 & DH-mGGA & 50 & 13 & 90,92 \\
B2-PLYP & 2006 & DH-GGA & 53 & 27 & 40 \\
mPW2-PLYP & 2006 & DH-GGA & 55 & 25 & 93 \\
B2GP-PLYP & 2008 & DH-GGA & 55 & 36 & 94 \\
ROB2-PLYP & 2009 & DH-GGA & 59 & 28 & 95 \\
B2 $\pi$-PLYP & 2009 & DH-GGA & 60 & 27 & 96 \\
B2T-PLYP & 2008 & DH-GGA & 60 & 31 & 94 \\
B2IP-PLYP & 2016 & DH-GGA & 60 & 35 & 97 \\
PBE-QIDH & 2014 & DH-GGA & 69 & 33 & 98 \\
TPSS-QIDH & 2014 & DH-mGGA & 69 & 33 & 98 \\
B2K-PLYP & 2008 & DH-GGA & 72 & 42 & 99 \\
mPW2K-PLYP & 2008 & DH-GGA & 72 & 42 & 99 \\
PBE0-2 & 2012 & DH-GGA & 79 & 50 & 100 \\
CIS(D) & 1994 & WFT & 100 & 100 & 47 \\
${ }^{a}$ List of acronyms: & GGA: generalized gradient approximation, mGGA: meta-GGA, DH: double-hybrid, \\
WFT: wave function theory. & & &
\end{tabular}




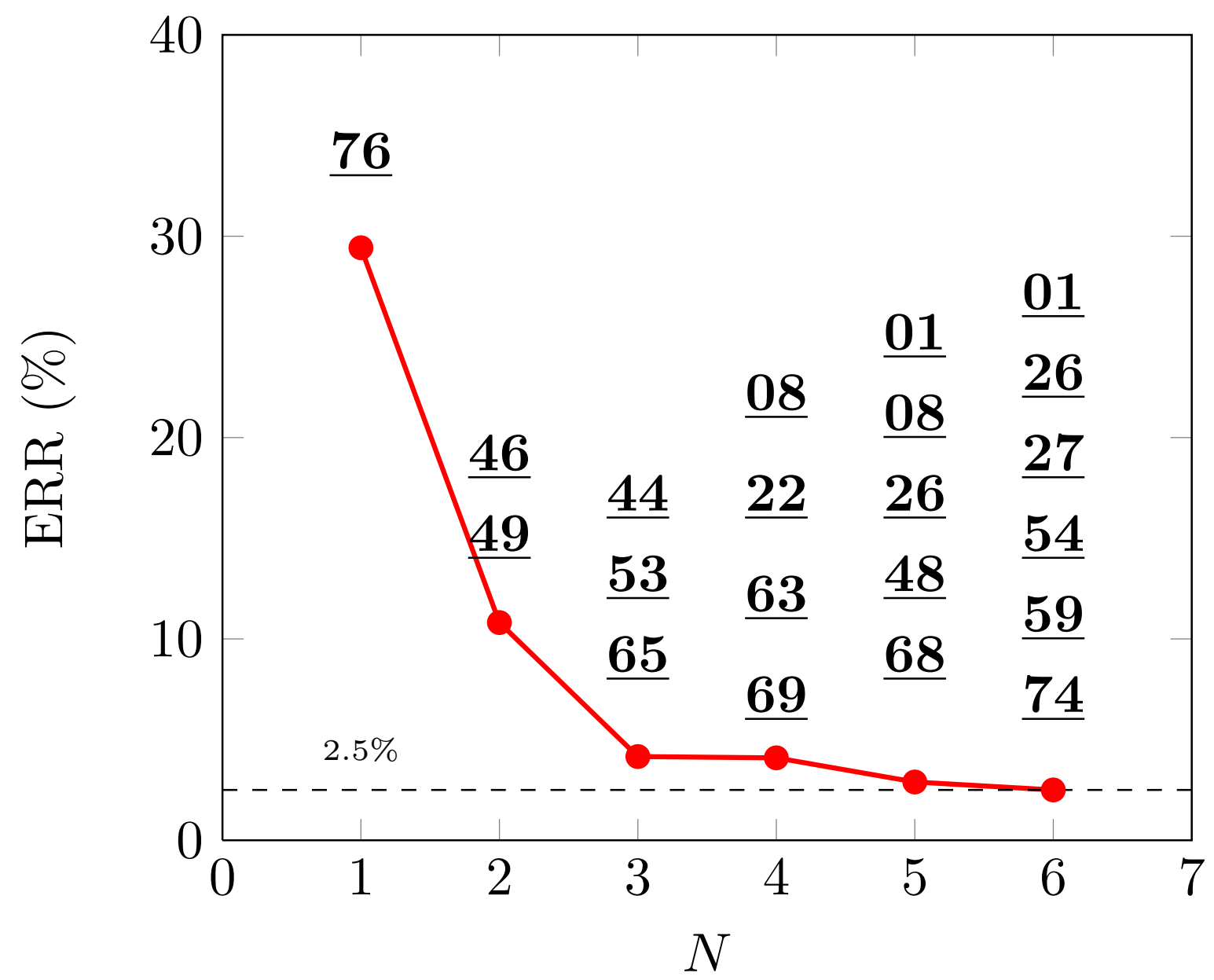

Figure 1: Evolution of the minimized error function (ERR; in \%) versus the number of systems $N$ in the EXN subset for a $2.5 \%$ cost limitation. For each $N$, the label of the systems included into the subset are added. The ERR function is estimated to $2.5 \%$ for the EX6-0 subset. A representation of all the systems is given in Figure S1 in the Supporting Information. 

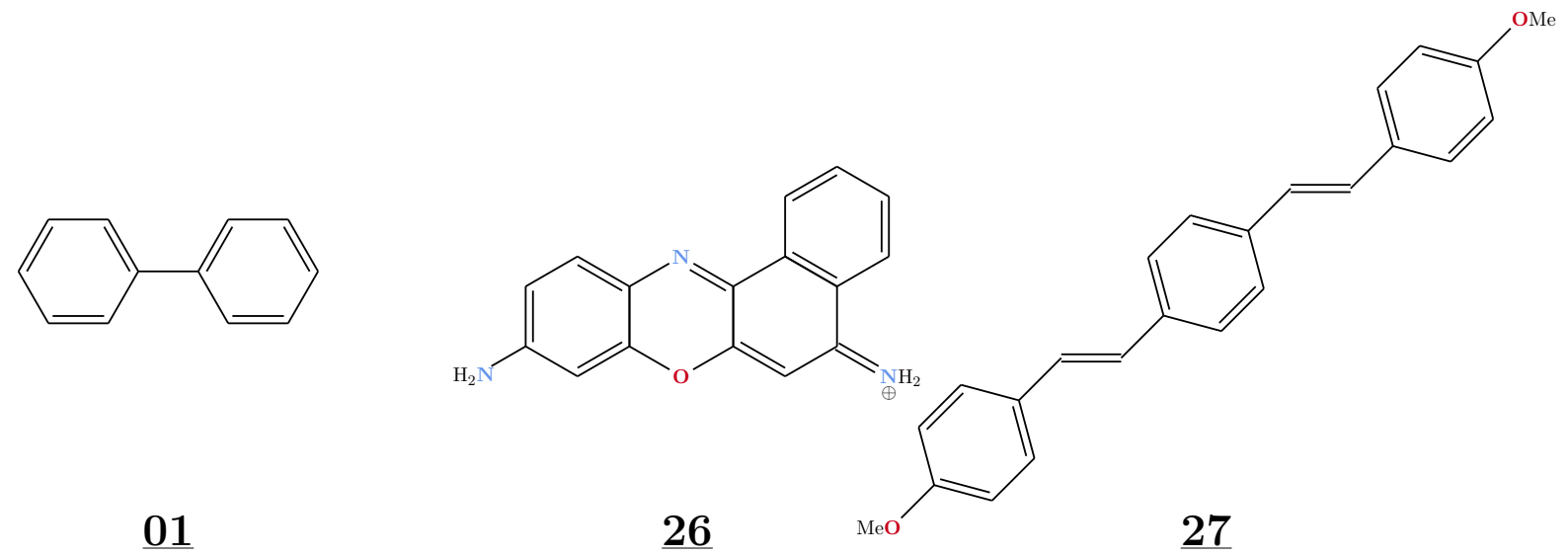<smiles>O=C1C2=C3c4sccc4C=CN3C(=O)C2=C2c3sccc3C=CN12</smiles>

$\underline{54}$<smiles>CC1(C)Oc2c(c3cccc4c(C#N)c(C#N)c5cccc2c5c43)O1</smiles>

$\underline{59}$<smiles>c1ccc(N(c2ccccc2)c2ccc(-c3cncs3)cc2)cc1</smiles>

74

Figure 2: Systems included into the EX6-0 subset. 


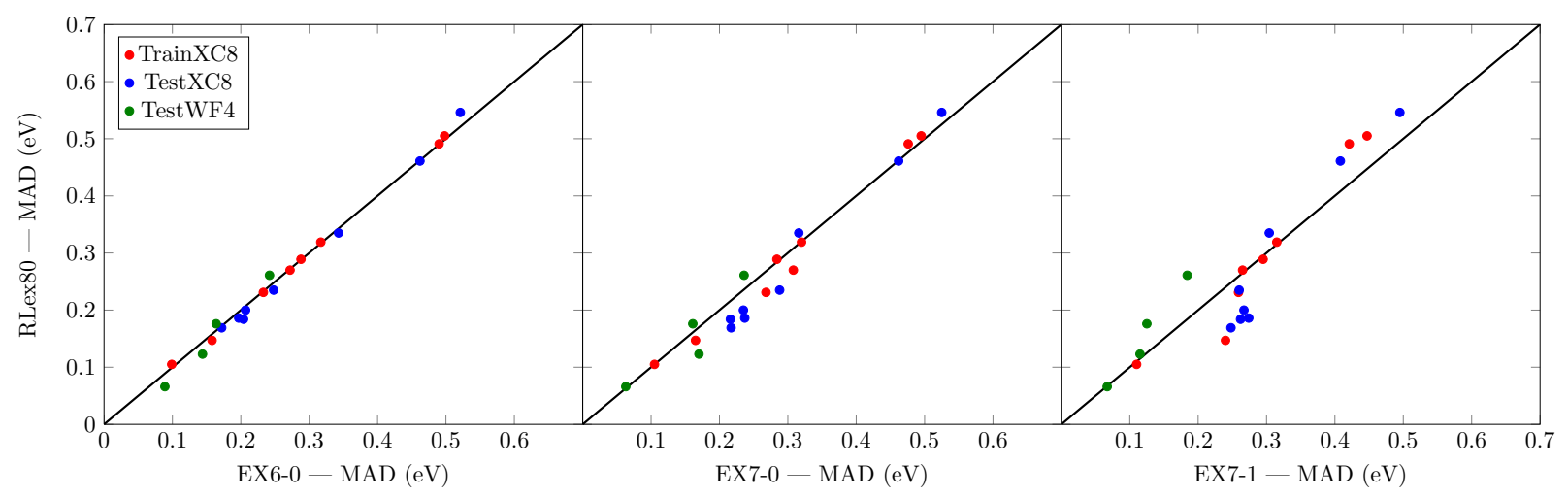

Figure 3: Correlations between the mean absolute deviation (MAD; in eV) statistical measure calculated on the large RLex80 dataset and its (left) EX6-0, (middle) EX7-0 and (right) EX7-1 subsets for the computational protocols part of the TrainXC8 training set (red), and the TestXC8 (blue) and TestWF4 (green) test sets. The MADs derive from the adiabatic energies computed with a density functional with respect to the CC2 adiabatic energies. More details about the training and test sets are given in Table S1 in the Supporting Information. 


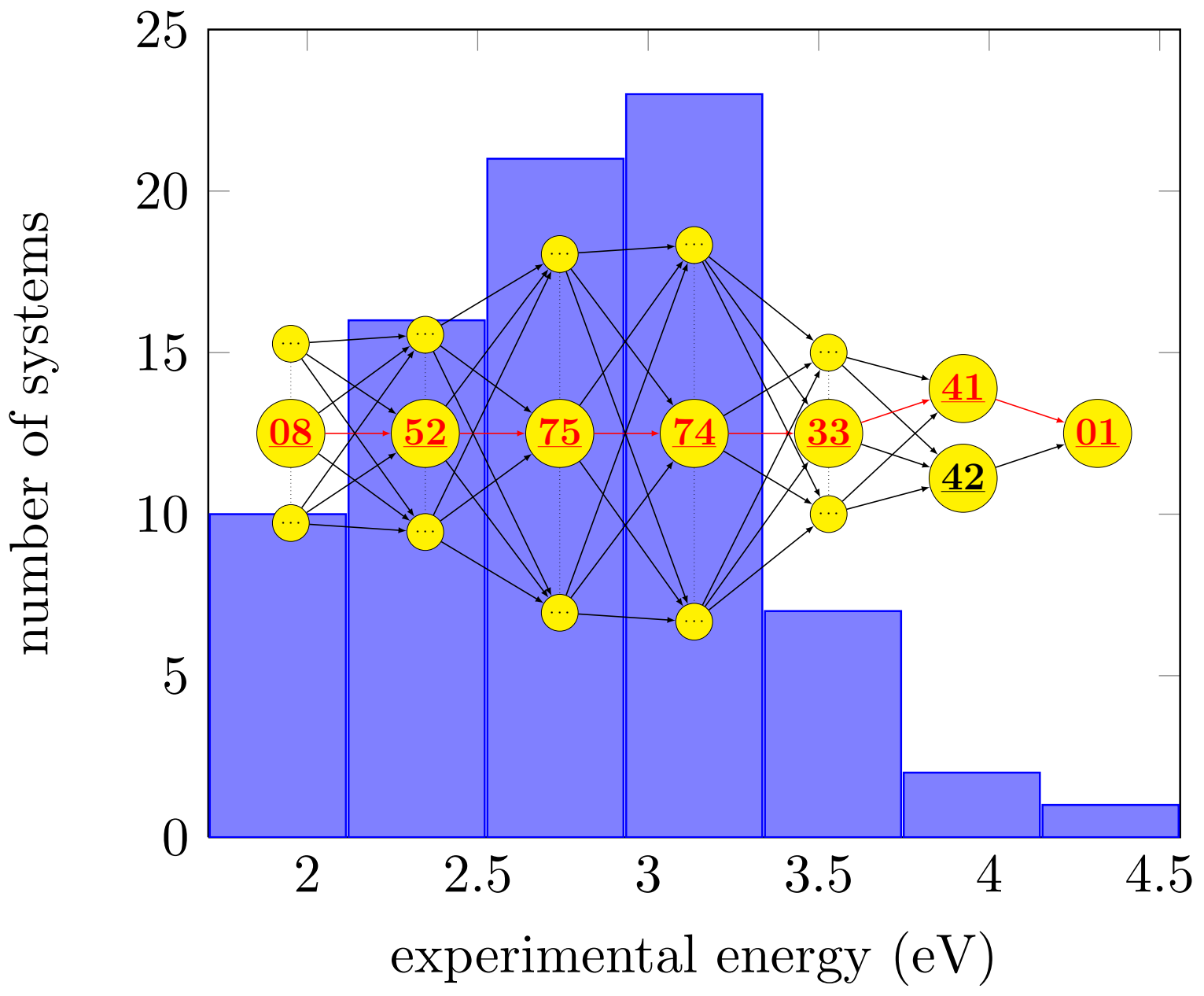

Figure 4: 1D-histogram displaying the range of experimental energies $(\mathrm{eV})$ in case of the RLex80 dataset. The overlayed decision diagram represents the chosen system in each bin to minimize the ERR function $(\mathrm{ERR}=5.7 \%)$ and to build the EX7-0 subset. A representation of all the systems is given in Figure S1 in the Supporting Information. 
<smiles>CCN([Tl])c1ccc(N([TlH])CC)c2c1C(=O)c1ccccc1C2=O</smiles>

08<smiles>c1ccc(C2CCC(C(c3ccccc3)c3ccccc3C3CCCC3)CC2)cc1</smiles>

74

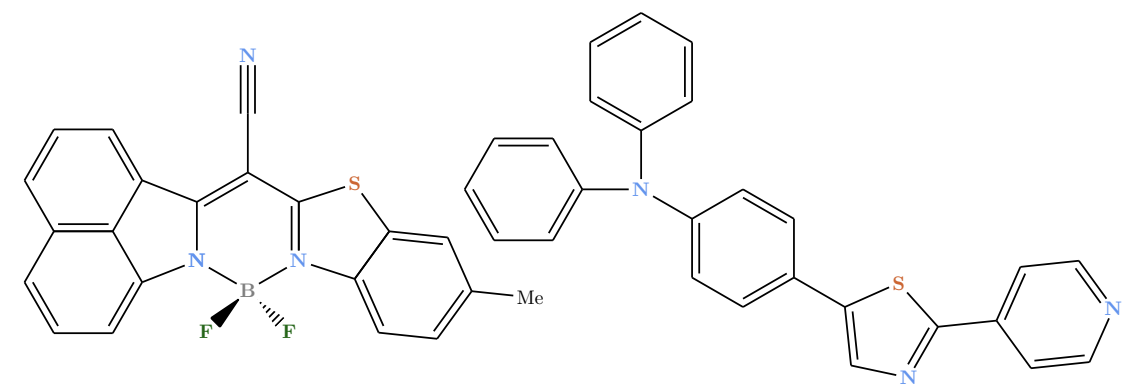

52<smiles></smiles>

33

41

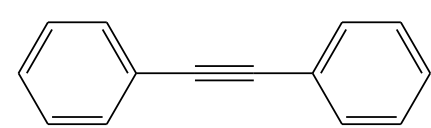

75

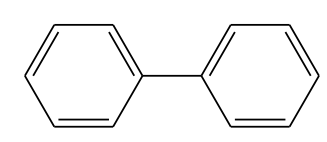

01 

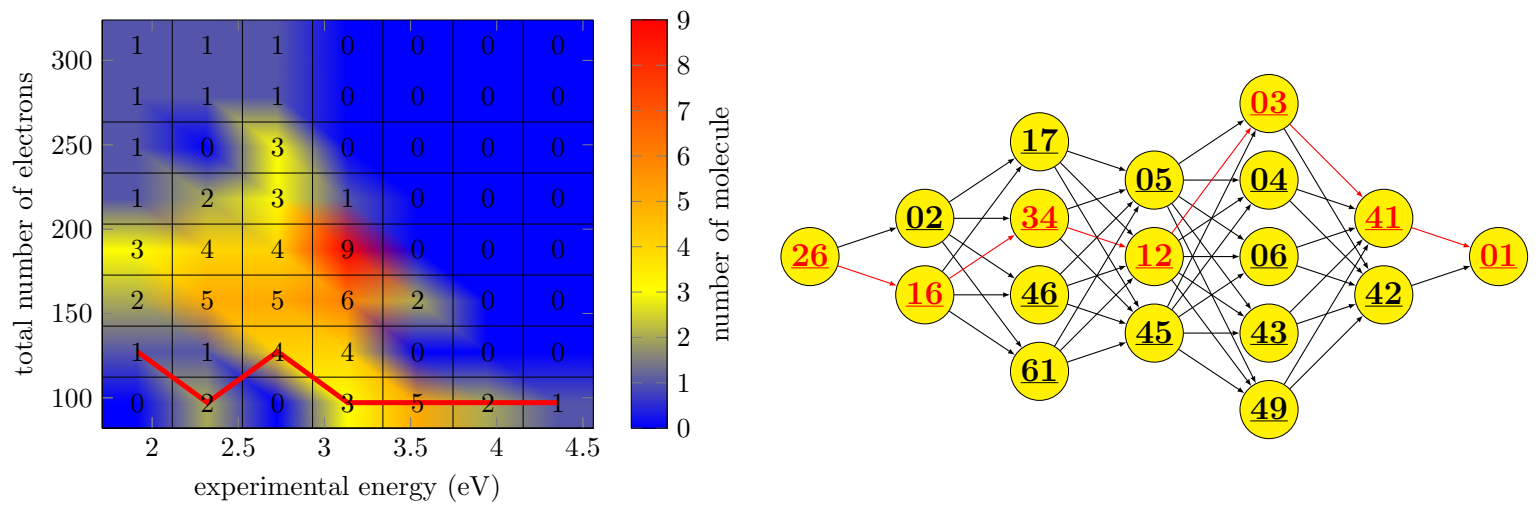

Figure 6: (left) 2D-histogram displaying the range of total number of electrons per system versus the range of experimental energies (eV) in case of the RLex80 dataset. (right) Decision diagram showing the chosen system in each 2D-bin roamed by the red line depicted on the 2D-histogram to minimize the ERR function $(\mathrm{ERR}=17.4 \%)$ and to build the EX7-1 subset. A representation of all the systems is given in Figure S1 in the Supporting Information. 
<smiles>Nc1ccc2nc3c4ccccc4c(=[W])cc-3oc2c1</smiles>

$\underline{26}$

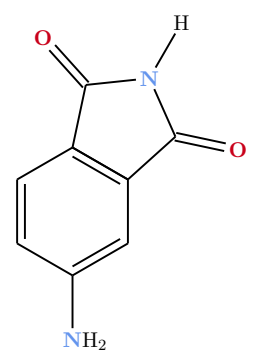

12<smiles>c1cc[n+](-c2nc3ccccc3[nH]2)cc1</smiles>

16<smiles>COc1ccc2ccc(=O)oc2c1</smiles>

$\underline{03}$

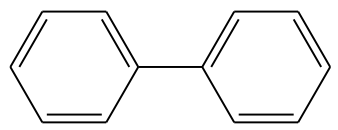

01

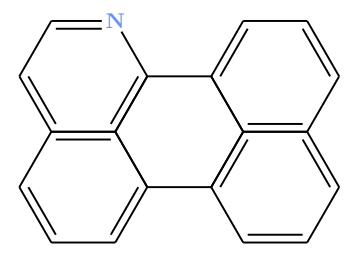

$\underline{34}$

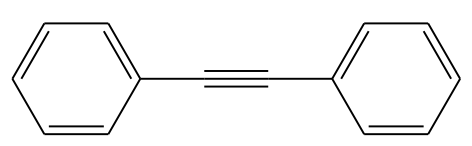

41

Figure 7: Systems included into the EX7-1 subset. 


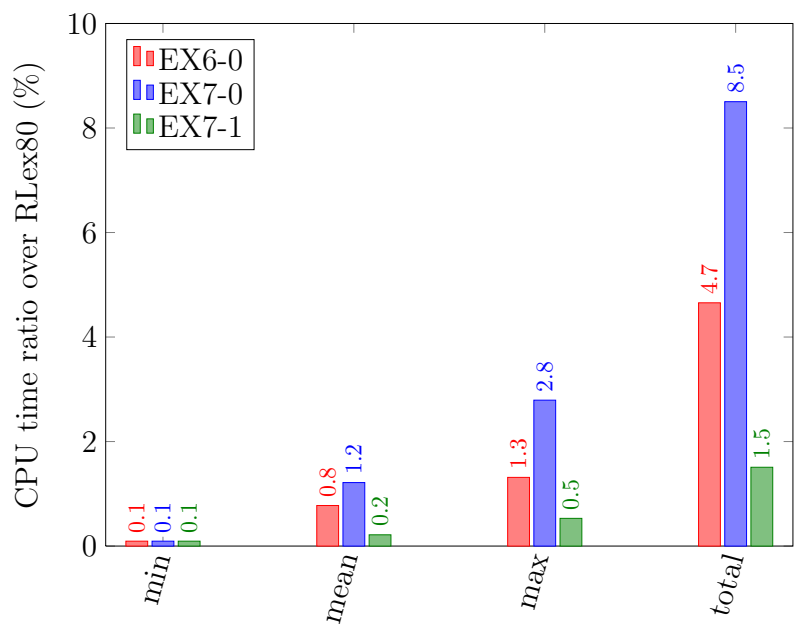

Figure 8: CPU time ratio (\%) with respect to the total CPU time to perform the computations of the $80+80$ TDDFT single-point energies of the RLex80 dataset $(10,689,674$ s). min and max labels refer to the minimum and maximum CPU times spent to perform the shortest and longest jobs, respectively, of the EX6-0, EX7-0 and EX7-1 subsets. The mean and total labels refer to the average and total CPU times, respectively, spent to perform the computations of each subset. Computations are performed on a 16 core $\operatorname{Intel}(\mathrm{R})$ Xeon(R) CPU E7-4830@2.13GHz CPU with the Orca software and the protocol is set to $\mathrm{PBE}-\mathrm{QIDH} /$ def2-TZVP. 


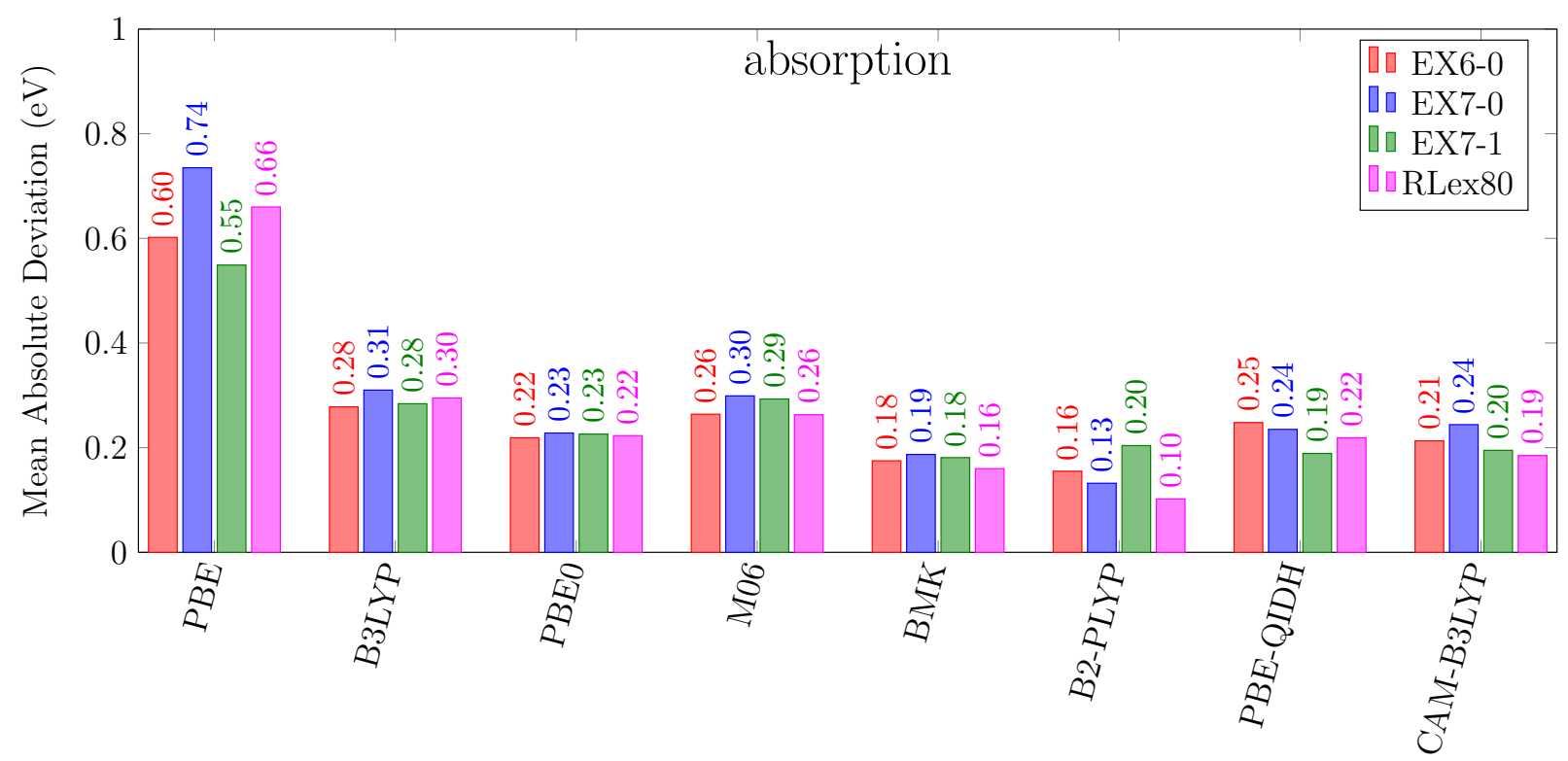

Figure 9: Mean absolute deviations (MAD; in $\mathrm{eV}$ ) of vertical absorption energies computed with a selection of semilocal, hybrid and double-hybrid density functionals for the RLex80 dataset and its EX6-0, EX7-0, EX7-1 subsets. All density functionals are ranked according to their fraction of exact-like exchange. B2-PLYP and PBE-QIDH computations are done with the def2-TZVP basis set, all others are done at the $6-31+\mathrm{G}(d)$ level of theory, and the CC2 method is taken as reference. 


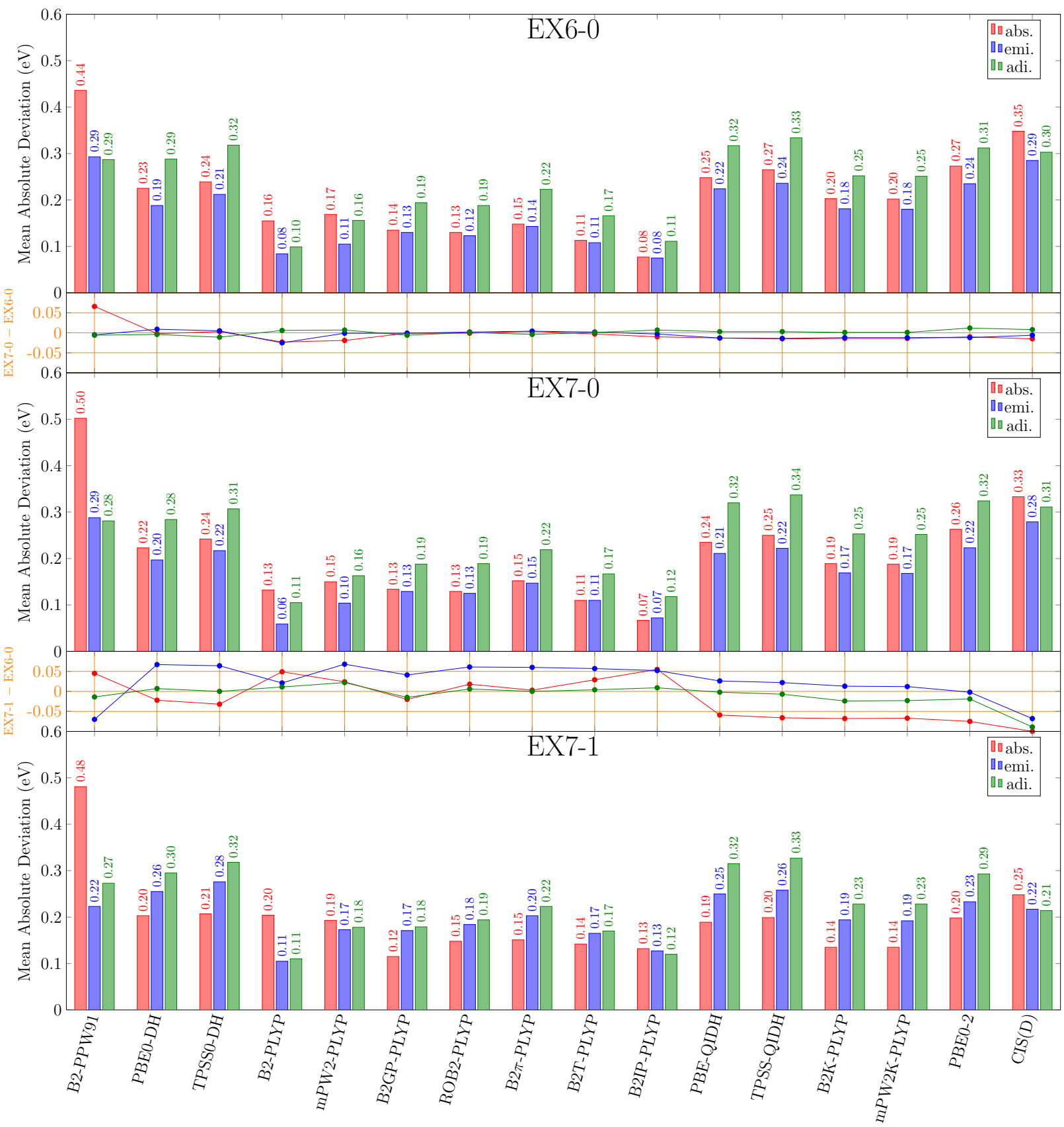

Figure 10: Mean absolute deviations (bar plots; eV) of vertical absorption, vertical emission and adiabatic energies computed with a selection of double-hybrid density functionals for the (top) EX6-0, (center) EX7-0 and (bottom) EX7-1 subsets. Scatter plots represent the difference between mean absolute deviations calculated from the (top) EX7-0 and EX6-0, (bottom) EX7-1 and EX6-0 subsets. All the computations are done with the def2-TZVP basis set, and the CC2 method is taken as reference. 


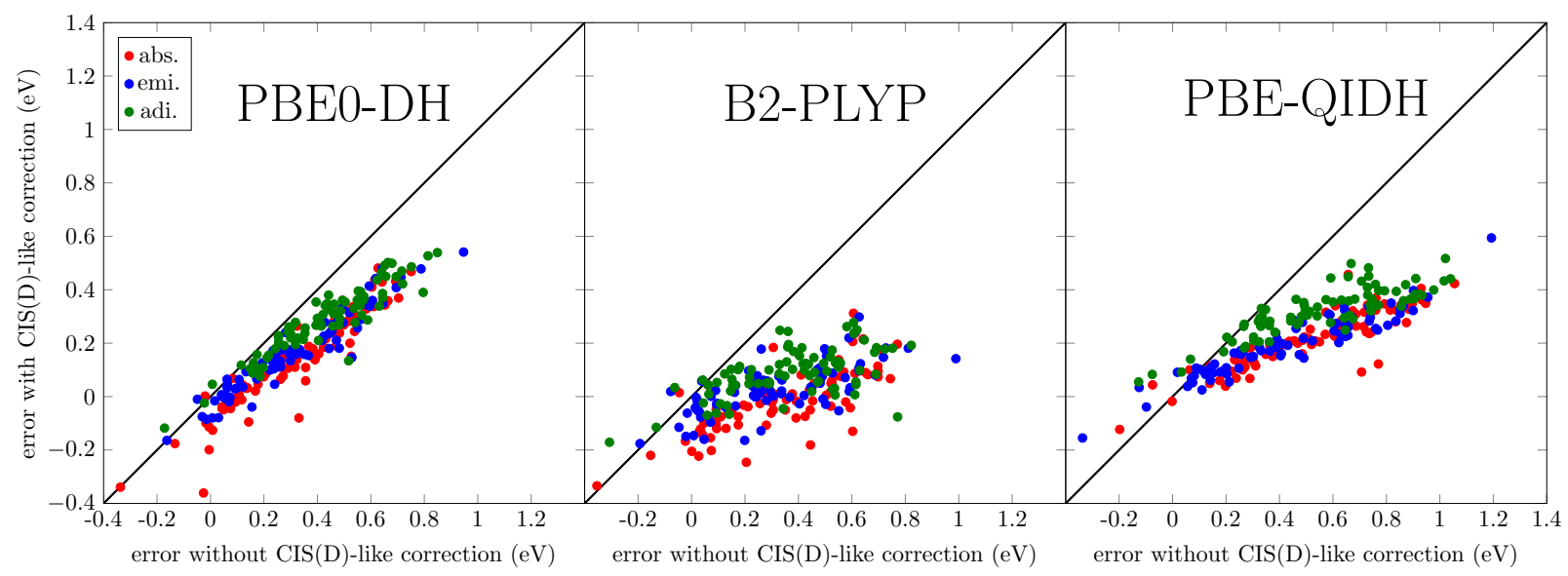

Figure 11: Correlations between the transition energy errors (red, vertical absorption; blue, vertical emission; green, adiabatic; in eV) computed with and without the CIS(D)-like correction for the (left) PBE0-DH, (center) B2-PLYP and (right) PBE-QIDH double-hybrid density functionals in case of the RLex80 dataset. For points positioned under the black diagonal line, the CIS(D)-like correction improves the estimation of the transition energy. All the computations are done with the def2-TZVP basis set, and the CC2 method is taken as reference. 


\section{Supporting Information Available}

A list of the computational protococols included into the TrainXC8, TestXC8 and TestWF4 sets is reported in Table S1. A sketch of the 80 large organic systems under investigation into the RLex80 dataset are depicted in Figure S1. Density-functional error trends as a function of the reference energy excitations are plotted in Figure S2. A basis set convergence test to compute vertical and adiabatic transitions with double-hybrid density functionals is reported in Figure S3. Correlations plots between the MSD, MAD and STD statistical measures calculated from the large RLex80 dataset and its EX6-0, EX7-0 and EX7-1 subsets for the computational protocols included into the training, test and the post-Hartree-Fock sets are reported in Figures S4, S5 and S6, respectively. Detailed statistical comparisons between MADs computed on the RLex80 dataset and its EX6-0, EX7-0 and EX7-1 subsets for the computational protocols included into the training, test and the post-Hartree-Fock sets are reported in Figures S7, S8 and S9, respectively.

\section{References}

(1) Robb, M. A.; Garavelli, M.; Olivucci, M.; Bernardi, F. Reviews in Computational Chemistry; 2007; pp 87-146.

(2) Savarese, M.; Brémond, E.; Antonov, L.; Ciofini, I.; Adamo, C. Computational Insights into Excited-State Proton-Transfer Reactions in Azo and Azomethine Dyes. ChemPhysChem 2015, 16, 3966-3973.

(3) Barone, V.; Bloino, J.; Biczysko, M.; Santoro, F. Fully Integrated Approach to Compute Vibrationally Resolved Optical Spectra: From Small Molecules to Macrosystems. J. Chem. Theory Comput. 2009, 5, 540-554.

(4) Brémond, E.; Alberto, M. E.; Russo, N.; Ricci, G.; Ciofini, I.; Adamo, C. Photophysical 
properties of NIR-emitting fluorescence probes: insights from TD-DFT. Phys. Chem. Chem. Phys. 2013, 15, 10019-10027.

(5) Hammes-Schiffer, S. Theory of Proton-Coupled Electron Transfer in Energy Conversion Processes. Acc. Chem. Res. 2009, 42, 1881-1889.

(6) Savarese, M.; Raucci, U.; Netti, P. A.; Adamo, C.; Ciofini, I.; Rega, N. Modeling of charge transfer processes to understand photophysical signatures: The case of Rhodamine 110. Chem. Phys. Lett. 2014, 610-611, 148-152.

(7) Antonov, L.; Deneva, V.; Simeonov, S.; Kurteva, V.; Crochet, A.; Fromm, K. M.; Shivachev, B.; Nikolova, R.; Savarese, M.; Adamo, C. Controlled Tautomeric Switching in Azonaphthols Tuned by Substituents on the Phenyl Ring. ChemPhysChem 2015, $16,649-657$.

(8) Laurent, A. D.; Adamo, C.; Jacquemin, D. Dye chemistry with time-dependent density functional theory. Phys. Chem. Chem. Phys. 2014, 16, 14334-14356.

(9) Di Tommaso, S.; Bousquet, D.; Moulin, D.; Baltenneck, F.; Riva, P.; David, H.; Fadli, A.; Gomar, J.; Ciofini, I.; Adamo, C. Theoretical approaches for predicting the color of rigid dyes in solution. J. Comput. Chem. 2017, 38, 998-1004.

(10) Jacquemin, D.; Brémond, E.; Ciofini, I.; Adamo, C. Impact of Vibronic Couplings on Perceived Colors: Two Anthraquinones as a Working Example. J. Phys. Chem. Lett. 2012, 3, 468-471.

(11) De Angelis, F.; Santoro, F.; Nazeruddin, M. K.; Barone, V. Ab Initio Prediction of the Emission Color in Phosphorescent Iridium(III) Complexes for OLEDs. J. Phys. Chem. B 2008, 112, 13181-13183.

(12) Brémond, E.; Bahers, T. L.; Ricci, G.; Ciofini, I.; Adamo, C. In silico assessment of the HPLC-UV response coefficients. Comput. Theor. Chem. 2014, 1040-1041, 1-5. 
(13) Brémond, E.; Ciofini, I.; Labat, F.; Ricci, G.; Adamo, C. Simulations of UV-visible spectra for analytical applications: phenothiazines as a case study. Mol. Sim. 2014, 40, 169-175.

(14) Hohenberg, P.; Kohn, W. Inhomogeneous Electron Gas. Phys. Rev. 1964, 136, B864B871.

(15) Casida, M. E. Recent Advances in Density Functional Methods; Chong, D. P., Ed.: World Scientific: Singapore, 1995; Vol. 1; pp 155-192.

(16) Brémond, E.; Ciofini, I.; Sancho-García, J. C.; Adamo, C. Nonempirical DoubleHybrid Functionals: An Effective Tool for Chemists. Acc. Chem. Res. 2016, 49, 1503-1513.

(17) Leang, S. S.; Zahariev, F.; Gordon, M. S. Benchmarking the performance of timedependent density functional methods. J. Chem. Phys. 2012, 136, 104101.

(18) Schreiber, M.; Silva-Junior, M. R.; Sauer, S. P. A.; Thiel, W. Benchmarks for electronically excited states: CASPT2, CC2, CCSD, and CC3. J. Chem. Phys. 2008, 128, 134110.

(19) Peach, M. J. G.; Benfield, P.; Helgaker, T.; Tozer, D. J. Excitation energies in density functional theory: An evaluation and a diagnostic test. J. Chem. Phys. 2008, 128, 044118 .

(20) Zhao, Y.; Truhlar, D. G. Density Functional for Spectroscopy: No Long-Range SelfInteraction Error, Good Performance for Rydberg and Charge-Transfer States, and Better Performance on Average than B3LYP for Ground States. J. Phys. Chem. A 2006, 110, 13126-13130.

(21) Goerigk, L.; Grimme, S. Assessment of TD-DFT methods and of various spin scaled 
CIS(D) and CC2 versions for the treatment of low-lying valence excitations of large organic dyes. J. Chem. Phys. 2010, 132, 184103.

(22) Goerigk, L.; Moellmann, J.; Grimme, S. Computation of accurate excitation energies for large organic molecules with double-hybrid density functionals. Phys. Chem. Chem. Phys. 2009, 11, 4611-4620.

(23) Jacquemin, D.; Wathelet, V.; Perpète, E. A.; Adamo, C. Extensive TD-DFT Benchmark: Singlet-Excited States of Organic Molecules. J. Chem. Theory Comput. 2009, 5, 2420-2435.

(24) Jacquemin, D.; Perpète, E. A.; Ciofini, I.; Adamo, C. Assessment of Functionals for TD-DFT Calculations of Singlet-Triplet Transitions. J. Chem. Theory Comput. 2010, $6,1532-1537$.

(25) Jacquemin, D.; Duchemin, I.; Blase, X. 0-0 Energies Using Hybrid Schemes: Benchmarks of TD-DFT, CIS(D), ADC(2), CC2, and BSE/GW formalisms for 80 Real-Life Compounds. J. Chem. Theory Comput. 2015, 11, 5340-5359.

(26) Fang, C.; Oruganti, B.; Durbeej, B. How Method-Dependent Are Calculated Differences between Vertical, Adiabatic, and 0-0 Excitation Energies? J. Phys. Chem. A 2014, 118, 4157-4171.

(27) Winter, N. O. C.; Graf, N. K.; Leutwyler, S.; Hattig, C. Benchmarks for 0-0 transitions of aromatic organic molecules: DFT/B3LYP, ADC(2), CC2, SOS-CC2 and SCS-CC2 compared to high-resolution gas-phase data. Phys. Chem. Chem. Phys. 2013, 15, $6623-6630$.

(28) Jacquemin, D.; Planchat, A.; Adamo, C.; Mennucci, B. TD-DFT Assessment of Functionals for Optical 0-0 Transitions in Solvated Dyes. J. Chem. Theory Comput. 2012, $8,2359-2372$. 
(29) Send, R.; Kühn, M.; Furche, F. Assessing Excited State Methods by Adiabatic Excitation Energies. J. Chem. Theory Comput. 2011, 7, 2376-2386.

(30) Savarese, M.; Raucci, U.; Fukuda, R.; Adamo, C.; Ehara, M.; Rega, N.; Ciofini, I. Comparing the performance of TD-DFT and SAC-CI methods in the description of excited states potential energy surfaces: An excited state proton transfer reaction as case study. J. Comput. Chem. 2017, 38, 1084-1092.

(31) Gozem, S.; Melaccio, F.; Valentini, A.; Filatov, M.; Huix-Rotllant, M.; Ferré, N.; Frutos, L. M.; Angeli, C.; Krylov, A. I.; Granovsky, A. A.; Lindh, R.; Olivucci, M. Shape of Multireference, Equation-of-Motion Coupled-Cluster, and Density Functional Theory Potential Energy Surfaces at a Conical Intersection. J. Chem. Theory Comput. 2014, 10, 3074-3084.

(32) Filatov, M. Assessment of Density Functional Methods for Obtaining Geometries at Conical Intersections in Organic Molecules. J. Chem. Theory Comput. 2013, 9, 45264541.

(33) Lynch, B. J.; Truhlar, D. G. Small Representative Benchmarks for Thermochemical Calculations. J. Phys. Chem. A 2003, 107, 8996-8999.

(34) Schultz, N. E.; Zhao, Y.; Truhlar, D. G. Databases for Transition Element Bonding: Metal-Metal Bond Energies and Bond Lengths and Their Use To Test Hybrid, Hybrid Meta, and Meta Density Functionals and Generalized Gradient Approximations. J. Phys. Chem. A 2005, 109, 4388-4403.

(35) Schultz, N. E.; Zhao, Y.; Truhlar, D. G. Density Functionals for Inorganometallic and Organometallic Chemistry. J. Phys. Chem. A 2005, 109, 11127-11143.

(36) Zheng, J.; Zhao, Y.; Truhlar, D. G. The DBH24/08 Database and Its Use to Assess Electronic Structure Model Chemistries for Chemical Reaction Barrier Heights. J. Chem. Theory Comput. 2009, 5, 808-821. 
(37) Di Meo, F.; Trouillas, P.; Adamo, C.; Sancho-García, J. C. Application of recent double-hybrid density functionals to low-lying singlet-singlet excitation energies of large organic compounds. The J. Chem. Phys. 2013, 139, 164104.

(38) Goerigk, L.; Grimme, S. Calculation of Electronic Circular Dichroism Spectra with Time-Dependent Double-Hybrid Density Functional Theory. J. Phys. Chem. A 2009, 113, 767-776.

(39) Grimme, S.; Neese, F. Double-hybrid density functional theory for excited electronic states of molecules. J. Chem. Phys. 2007, 127, 154116.

(40) Grimme, S. Semiempirical hybrid density functional with perturbative second-order correlation. J. Chem. Phys. 2006, 124, 034108.

(41) Su, N. Q.; Xu, X. The XYG3 type of doubly hybrid density functionals. Wiley Interdiscip. Rev. Comput. Mol. Sci. 2016, 6, 721-747.

(42) Goerigk, L.; Grimme, S. Double-hybrid density functionals. Wiley Interdiscip. Rev. Comput. Mol. Sci. 2014, 4, 576-600.

(43) Sancho-Garcia, J. C.; Adamo, C. Double-hybrid density functionals: merging wavefunction and density approaches to get the best of both worlds. Phys. Chem. Chem. Phys. 2013, 15, 14581-14594.

(44) Bousquet, D.; Brémond, E.; Sancho-García, J. C.; Ciofini, I.; Adamo, C. Is There Still Room for Parameter Free Double Hybrids? Performances of PBE0-DH and B2PLYP over Extended Benchmark Sets. J. Chem. Theory Comput. 2013, 9, 3444-3452.

(45) Brémond, E.; Savarese, M.; Sancho-García, J. C.; Pérez-Jiménez, A. J.; Adamo, C. Systematic Improvement of Density Functionals through Parameter-Free Hybridization Schemes. J. Phys. Chem. Lett. 2015, 6, 3540-3545. 
(46) Hirata, S.; Head-Gordon, M. Time-dependent density functional theory within the Tamm-Dancoff approximation. Chem. Phys. Lett. 1999, 314, 291-299.

(47) Head-Gordon, M.; Rico, R. J.; Oumi, M.; Lee, T. J. A doubles correction to electronic excited states from configuration interaction in the space of single substitutions. Chem. Phys. Lett. 1994, 219, 21-29.

(48) Civalleri, B.; Presti, D.; Dovesi, R.; Savin, A. Chemical Modelling: Applications and Theory Volume 9; The Royal Society of Chemistry, 2012; Vol. 9; pp 168-185.

(49) Freedman, D.; Diaconis, P. On the histogram as a density estimator: $L_{2}$ theory. $Z$. Wahrscheinlichkeit 1981, 57, 453-476.

(50) Perdew, J. P.; Burke, K.; Ernzerhof, M. Generalized gradient approximation made simple. Phys. Rev. Lett. 1996, 77, 3865-3868.

(51) Adamo, C.; Barone, V. Toward reliable density functional methods without adjustable parameters: The PBE0 model. J. Chem. Phys. 1999, 110, 6158-6170.

(52) Ernzerhof, M.; Scuseria, G. E. Assessment of the Perdew-Burke-Ernzerhof exchangecorrelation functional. J. Chem. Phys. 1999, 110, 5029-5036.

(53) Zhao, Y.; Schultz, N. E.; Truhlar, D. G. The M06 suite of density functionals for main group thermochemistry, thermochemical kinetics, noncovalent interactions, excited states, and transition elements: two new functionals and systematic testing of four M06-class functionals and 12 other functionals. Theor. Chem. Acc. 2008, 120, 215241.

(54) Vydrov, O. A.; Scuseria, G. E. Assessment of a long range corrected hybrid functional. J. Chem. Phys. 2006, 125, 234109.

(55) Vydrov, O. A.; Heyd, J.; Krukau, A.; Scuseria, G. E. Importance of short-range versus 
long-range Hartree-Fock exchange for the performance of hybrid density functionals. J. Chem. Phys. 2006, 125, 074106.

(56) Vydrov, O. A.; Scuseria, G. E.; Perdew, J. P. Tests of functionals for systems with fractional electron number. J. Chem. Phys. 2007, 126, 154109.

(57) Chai, J.-D.; Head-Gordon, M. Long-range corrected hybrid density functionals with damped atom-atom dispersion corrections. Phys. Chem. Chem. Phys. 2008, 10, 66156620.

(58) Zhao, Y.; Truhlar, D. G. A new local density functional for main-group thermochemistry, transition metal bonding, thermochemical kinetics, and noncovalent interactions. J. Chem. Phys. 2006, 125, 194101.

(59) Peverati, R.; Truhlar, D. G. Exchange-Correlation Functional with Good Accuracy for Both Structural and Energetic Properties while Depending Only on the Density and Its Gradient. J. Chem. Theory Comput. 2012, 8, 2310-2319.

(60) Becke, A. D. Density-functional thermochemistry. III. The role of exact exchange. J. Chem. Phys. 1993, 98, 5648-5652.

(61) Barone, V.; Orlandini, L.; Adamo, C. Proton transfer in model hydrogen-bonded systems by a density functional approach. Chem. Phys. Lett. 1994, 231, 295-300.

(62) Stephens, P. J.; Devlin, F. J.; Frisch, M. J.; Chabalowski, C. F. Ab initio Calculation of Vibrational Absorption and Circular Dichroism Spectra Using Density Functional Force Fields. J. Phys. Chem. 1994, 98, 11623-11627.

(63) Boese, A. D.; Martin, J. M. L. Development of Density Functionals for Thermochemical Kinetics. J. Chem. Phys. 2004, 121, 3405-3416.

(64) Peverati, R.; Truhlar, D. G. Screened-exchange density functionals with broad accuracy for chemistry and solidstate physics. Phys. Chem. Chem. Phys. 2012, 14, 16187. 
(65) Yanai, T.; Tew, D.; Handy, N. A new hybrid exchange-correlation functional using the Coulomb-attenuating method (CAM-B3LYP). Chem. Phys. Lett. 2004, 393, 51-57.

(66) Zhao, Y.; Truhlar, D. G. Comparative DFT study of van der Waals complexes: Raregas dimers, alkaline-earth dimers, zinc dimer, and zinc-rare-gas dimers. J. Phys. Chem. 2006, 110, 5121-5129.

(67) Zhao, Y.; Truhlar, D. G. Density Functional for Spectroscopy: No Long-Range SelfInteraction Error, Good Performance for Rydberg and Charge-Transfer States, and Better Performance on Average than B3LYP for Ground States. J. Phys. Chem. A 2006, 110, 13126-13130.

(68) Hehre, W. J.; Ditchfield, R.; Pople, J. A. Self-Consistent Molecular Orbital Methods. XII. Further Extensions of Gaussian-Type Basis Sets for Use in Molecular Orbital Studies of Organic Molecules. J. Chem. Phys. 1972, 56, 2257-2261.

(69) Francl, M. M.; Pietro, W. J.; Hehre, W. J.; Binkley, J. S.; Gordon, M. S.; DeFrees, D. J.; Pople, J. A. Self-consistent molecular orbital methods. XXIII. A polarization-type basis set for second-row elements. J. Chem. Phys. 1982, 77, 36543665 .

(70) Ciofini, I.; Adamo, C. Accurate Evaluation of Valence and Low-Lying Rydberg States with Standard Time-Dependent Density Functional Theory. J. Phys. Chem. A 2007, $111,5549-5556$.

(71) Hay, P. J.; Wadt, W. R. Ab initio effective core potentials for molecular calculations. Potentials for the transition metal atoms Sc to Hg. J. Chem. Phys. 1985, 82, 270-283.

(72) Wadt, W. R.; Hay, P. J. Ab initio effective core potentials for molecular calculations. Potentials for main group elements Na to Bi. J. Chem. Phys. 1985, 82, 284-298. 
(73) Frisch, M. J.; Trucks, G. W.; Schlegel, H. B.; Scuseria, G. E.; Robb, M. A.; Cheeseman, J. R.; Scalmani, G.; Barone, V.; Mennucci, B.; Petersson, G. A.; Nakatsuji, H.; Caricato, M.; Li, X.; Hratchian, H. P.; Izmaylov, A. F.; Bloino, J.; Zheng, G.; Sonnenberg, J. L.; Hada, M.; Ehara, M.; Toyota, K.; Fukuda, R.; Hasegawa, J.; Ishida, M.; Nakajima, T.; Honda, Y.; Kitao, O.; Nakai, H.; Vreven, T.; Montgomery, J. A., Jr.; Peralta, J. E.; Ogliaro, F.; Bearpark, M.; Heyd, J. J.; Brothers, E.; Kudin, K. N.; Staroverov, V. N.; Kobayashi, R.; Normand, J.; Raghavachari, K.; Rendell, A.; Burant, J. C.; Iyengar, S. S.; Tomasi, J.; Cossi, M.; Rega, N.; Millam, J. M.; Klene, M.; Knox, J. E.; Cross, J. B.; Bakken, V.; Adamo, C.; Jaramillo, J.; Gomperts, R.; Stratmann, R. E.; Yazyev, O.; Austin, A. J.; Cammi, R.; Pomelli, C.; Ochterski, J. W.; Martin, R. L.; Morokuma, K.; Zakrzewski, V. G.; Voth, G. A.; Salvador, P.; Dannenberg, J. J.; Dapprich, S.; Daniels, A. D.; Farkas, Ö.; Foresman, J. B.; Ortiz, J. V.; Cioslowski, J.; Fox, D. J. Gaussian 09 Revision D.01. 2009; Gaussian Inc. Wallingford CT.

(74) Neese, F. The ORCA program system. WIREs Comput. Mol. Sci. 2012, 2, 73-78.

(75) Neese, F.; Wennmohs, F.; Hansen, A.; Becker, U. Efficient, approximate and parallel Hartree-Fock and hybrid DFT calculations. A 'chain-of-spheres' algorithm for the Hartree-Fock exchange. Chem. Phys. 2009, 356, 98-109.

(76) Rebolini, E.; Izsák, R.; Reine, S. S.; Helgaker, T.; Pedersen, T. B. Comparison of three efficient approximate exact-exchange algorithms: chain-of-spheres, pair-atomic resolution of the identity, and the auxiliary density matrix method. J. Chem. Theory Comput. 2016, 12, 3514-3522.

(77) Weigend, F.; Ahlrichs, R. Balanced basis sets of split valence, triple zeta valence and quadruple zeta valence quality for H to Rn: Design and assessment of accuracy. Phys. Chem. Chem. Phys. 2005, 7, 3297-3305. 
(78) Hellweg, A.; Hättig, C.; Höfener, S.; Klopper, W. Optimized accurate auxiliary basis sets for RI-MP2 and RI-CC2 calculations for the atoms Rb to Rn. Theor. Chem. Acc. 2007, 117, 587-597.

(79) Jr., T. H. D. Gaussian basis sets for use in correlated molecular calculations. I. The atoms boron through neon and hydrogen. J. Chem. Phys. 1989, 90, 1007-1023.

(80) Kendall, R. A.; Jr., T. H. D.; Harrison, R. J. Electron affinities of the first-row atoms revisited. Systematic basis sets and wave functions. J. Chem. Phys. 1992, 96, 67966806.

(81) Schirmer, J. Beyond the random-phase approximation: A new approximation scheme for the polarization propagator. Phys. Rev. A 1982, 26, 2395-2416.

(82) Hellweg, A.; Grun, S. A.; Hattig, C. Benchmarking the performance of spin-component scaled CC2 in ground and electronically excited states. Phys. Chem. Chem. Phys. 2008, 10, 4119-4127.

(83) Christiansen, O.; Koch, H.; Jørgensen, P. The second-order approximate coupled cluster singles and doubles model CC2. Chem. Phys. Lett. 1995, 243, 409-418.

(84) Hanke, W.; Sham, L. J. Many-Particle Effects in the Optical Excitations of a Semiconductor. Phys. Rev. Lett. 1979, 43, 387-390.

(85) Sham, L. J.; Rice, T. M. Many-Particle Derivation of the Effective-Mass Equation for the Wannier Exciton. Phys. Rev. 1966, 144, 708-714.

(86) Jacquemin, D.; Perpète, E. A.; Ciofini, I.; Adamo, C. Accurate simulation of optical properties in dyes. Acc. Chem. Res. 2009, 42, 326-334.

(87) Brémond, E.; Savarese, M.; Su, N. Q.; Pérez-Jiménez, Á. J.; Xu, X.; SanchoGarcía, J. C.; Adamo, C. Benchmarking Density Functionals on Structural Param- 
eters of Small-/Medium-Sized Organic Molecules. J. Chem. Theory Comput. 2016, 12, 459-465.

(88) Adamo, C.; Cossi, M.; Scalmani, G.; Barone, V. Accurate static polarizabilities by density functional theory: assessment of the PBE0 model. Chem. Phys. Lett. 1999, 307, 265-271.

(89) Mohajeri, A.; Alipour, M. B2-PPW91: A promising double-hybrid density functional for the electric response properties. J. Chem. Phys. 2012, 136, 124111.

(90) Brémond, E.; Adamo, C. Seeking for parameter-free double-hybrid functionals: The PBE0-DH model. J. Chem. Phys. 2011, 135, 024106.

(91) Toulouse, J.; Sharkas, K.; Brémond, E.; Adamo, C. Rationale for a new class of double-hybrid approximations in density-functional theory. J. Chem. Phys. 2011, 135, 101102.

(92) Aragó, J.; Ortí, E.; Sancho-García, J. C. Nonlocal van der Waals Approach Merged with Double-Hybrid Density Functionals: Toward the Accurate Treatment of Noncovalent Interactions. J. Chem. Theory Comput. 2013, 9, 3437-3443.

(93) Schwabe, T.; Grimme, S. Towards chemical accuracy for the thermodynamics of large molecules: new hybrid density functionals including non-local correlation effects. Phys. Chem. Chem. Phys. 2006, 8, 4398-4401.

(94) Karton, A.; Tarnopolsky, A.; Lamère, J.-F.; Schatz, G. C.; Martin, J. M. L. Highly Accurate First-Principles Benchmark Data Sets for the Parametrization and Validation of Density Functional and Other Approximate Methods. Derivation of a Robust, Generally Applicable, Double-Hybrid Functional for Thermochemistry and Thermochemical Kinetics. J. Phys. Chem. A 2008, 112, 12868-12886. 
(95) Graham, D. C.; Menon, A. S.; Goerigk, L.; Grimme, S.; Radom, L. Optimization and Basis-Set Dependence of a Restricted-Open-Shell Form of B2-PLYP Double-Hybrid Density Functional Theory. J. Phys. Chem. A 2009, 113, 9861-9873.

(96) Sancho-García, J. C.; Pérez-Jiménez, A. J. Assessment of double-hybrid energy functionals for $\pi$-conjugated systems. J. Chem. Phys. 2009, 131, 084108.

(97) Margraf, J. T.; Verma, P.; Bartlett, R. J. Ionization potential optimized double-hybrid density functional approximations. J. Chem. Phys. 2016, 145, 104106.

(98) Brémond, E.; Sancho-García, J. C.; Pérez-Jiménez, A. J.; Adamo, C. Double-hybrid functionals from adiabatic-connection: The QIDH model. J. Chem. Phys. 2014, 141, 031101-031104.

(99) Tarnopolsky, A.; Karton, A.; Sertchook, R.; Vuzman, D.; Martin, J. M. L. DoubleHybrid Functionals for Thermochemical Kinetics. J. Phys. Chem. A 2008, 112, 3-8.

(100) Chai, J.-D.; Mao, S.-P. Seeking for reliable double-hybrid density functionals without fitting parameters: The PBE0-2 functional. Chem. Phys. Lett. 2012, 538, 121-125. 


\section{Graphical TOC Entry}

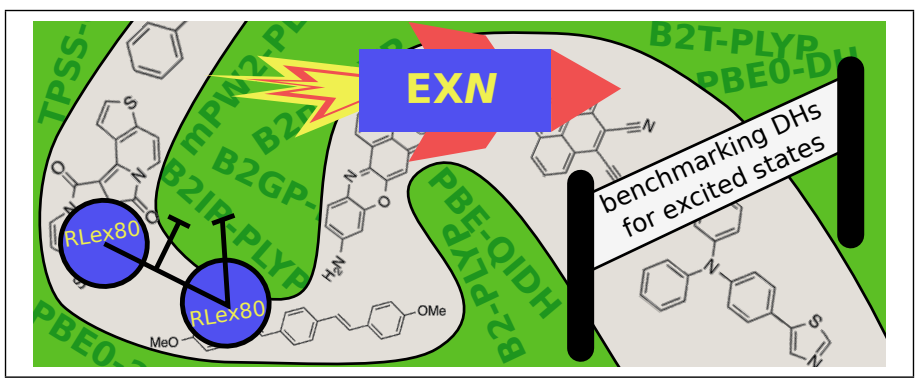

